\section{(6) OPEN ACCESS}

\title{
Systematic review and meta-analyses of risk factors for childhood overweight identifiable during infancy
}

\author{
Stephen Franklin Weng, ${ }^{1}$ Sarah A Redsell, ${ }^{2}$ Judy A Swift, ${ }^{3}$ Min Yang, ${ }^{4}$ \\ Cristine P Glazebrook ${ }^{5}$
}

- Additional appendices are published online only. To view these files please visit the journal online (http://dx.doi.org/ 10.1136/archdischild-2012302263).

${ }^{1}$ Division of Epidemiology and Public Health, School of Community Health Sciences, University of Nottingham,

Nottingham, UK

${ }^{2}$ School of Nursing, Midwifery and Physiotherapy, University of Nottingham, Queen's Medical Centre, Nottingham, UK ${ }^{3}$ Division of Nutritional Sciences, University of Nottingham, Sutton Bonington Campus, Loughborough, UK

${ }^{4}$ Nottingham Clinical Trials Unit, Nottingham Health Science

Partners, Queen's Medical Centre, Nottingham, UK

${ }^{5}$ Division of Psychiatry, Institute of Mental Health, University of Nottingham Innovation Park Nottingham, UK

\section{Correspondence to}

Professor Cris Glazebrook, Division of Psychiatry, Institute of Mental Health, University of Nottingham Innovation Park, Nottingham NG7 2TU, UK; Cris.Glazebrook@nottingham. ac.uk

Received 30 April 2012 Accepted 21 August 2012 Published Online First 29 October 2012

\section{ABSTRACT}

Objective To determine risk factors for childhood overweight that can be identified during the first year of life to facilitate early identification and targeted intervention.

Design Systematic review and meta-analysis.

Search strategy Electronic database search of MEDLINE, EMBASE, PubMed and CAB Abstracts.

Eligibility criteria Prospective observational studies following up children from birth for at least 2 years.

Results Thirty prospective studies were identified. Significant and strong independent associations with childhood overweight were identified for maternal prepregnancy overweight, high infant birth weight and rapid weight gain during the first year of life. Meta-analysis comparing breastfed with non-breastfed infants found a $15 \%$ decrease $\left(95 \% \mathrm{Cl} 0.74\right.$ to $0.99 ;\left.\right|^{2}=73.3 \% ; n=10$ ) in the odds of childhood overweight. For children of mothers smoking during pregnancy there was a $47 \%$ increase $\left(95 \% \mathrm{Cl} 1.26\right.$ to $\left.1.73 ;\left.\right|^{2}=47.5 \% ; n=7\right)$ in the odds of childhood overweight. There was some evidence associating early introduction of solid foods and childhood overweight. There was conflicting evidence for duration of breastfeeding, socioeconomic status at birth, parity and maternal marital status at birth. No association with childhood overweight was found for maternal age or education at birth, maternal depression or infant ethnicity. There was inconclusive evidence for delivery type, gestational weight gain, maternal postpartum weight loss and 'fussy' infant temperament due to the limited number of studies.

Conclusions Several risk factors for both overweight and obesity in childhood are identifiable during infancy. Future research needs to focus on whether it is clinically feasible for healthcare professionals to identify infants at greatest risk.

\section{INTRODUCTION}

In the UK in $2008,31 \%$ of boys and $29 \%$ of girls aged 2-15 years were classified as overweight or obese. ${ }^{1}$ These figures are supported by data collected in 2010 by the National Child Measurement Programme showing that $23 \%$ of children aged $4-5$ years and $33 \%$ of $10-11$ year olds are overweight. $^{2}$ Evidence suggests that weight at 5 years of age is a good indicator of the future health of a child $^{3}$ and that obesity during childhood increases the risk of adult obesity. ${ }^{4}$ Cardiovascular disease, type 2 diabetes, obesity-attributable cancers, osteoarthritis and psychological disturbance generate much of the morbidity and years of life lost associated with increasing levels of obesity. ${ }^{5}$

There is a strong rationale for intervening during early life in infants at risk of developing childhood

\section{What is already known on this topic}

- There is evidence that overweight or obesity during childhood increases the risk of adult obesity.

- Previous reviews have identified rapid weight gain, high birth weight and maternal smoking in pregnancy as important risk factors for childhood obesity.

\section{What this study adds}

- Early rapid weight gain, high birth weight, maternal pre-pregnancy overweight and maternal smoking in pregnancy increase the likelihood of childhood obesity and overweight.

- Breastfeeding and the late introduction of solid foods is moderately protective against childhood overweight.

- Other maternal and infant factors were not associated with childhood overweight.

obesity $^{6}$ and to date interventions have focused on nutritional modification through supporting parents regarding, for example, healthy eating and breastfeeding. $^{7-10}$ Both the Canadian Paediatric Society ${ }^{11}$ and the American Academy of Pediatrics ${ }^{12}$ advocate that all typically developing children aged 2 years and older should have their growth monitored to screen for under-development, wasting, overweight and obesity. However, in many countries, early life intervention is not routine clinical practice. Although in the USA, the Institute of Medicine has recently introduced early childhood obesity prevention guidance ${ }^{13}$ suggesting that healthcare professionals (HCPs) should undertake regular growth monitoring and consider obesity risk factors during infancy, there is evidence in both the UK and the USA that HCPs are reluctant to diagnose obesity in infants. ${ }^{14}$ Furthermore, HCPs who routinely provide advice to parents have been found to have low levels of knowledge concerning the risks of obesity. ${ }^{15}$ This is partly due to the implementation gap between published research and clinical practice. Currently, there have been no systematic reviews that have comprehensively investigated all infant risk factors for both overweight and obesity in childhood using only prospective studies. 
The aim of this systematic review is to identify those risk factors for overweight in childhood which could be identified by HCPs during an infant's first year of life.

\section{METHODS}

\section{Search strategy and data extraction}

MEDLINE, EMBASE, CAB Abstracts and PubMed articles published from 1990 to May 2011 were searched electronically. These dates were chosen because the identification of early life determinants of childhood overweight or obesity is a relatively recent area of research interest. Keywords, identified in the literature and through group discussion on early life risk factors for childhood overweight, were used to search for all relevant publications and are given in online supplementary appendix 1. One reviewer (SFW) screened studies based on titles and abstracts. Subsequently, full-text articles were screened and independently selected for inclusion in the systematic review by two authors (SFW and SAR; SFW and JAS; or SFW and CG) based on specific eligibility criteria. The reviewers independently assessed the methodological quality of the papers using the Newcastle-Ottawa Scale. ${ }^{16}$ This assessment takes into account epidemiological quality in relation to control of confounding variables, adequate sample size, minimisation of selection bias and clear definitions of exposures. Studies were judged according to: (i) selection of the study groups (scored 0-4); (ii) comparability of the study groups (scored 0-2); and (iii) ascertainment of the outcome (scored $0-3$ ). Data extraction and quality assessment forms are given in online supplementary appendix 2 .

\section{Study selection}

Studies were only considered if they were prospective studies and there was a minimum follow-up of 2 years from birth to allow for a diagnosis childhood overweight (as there is no standard definition of overweight in children under 2 years of age). ${ }^{17} 18$ We used 16 years of age as the cut-off for the follow-up of children. Although most children will have obtained close to their final height by 14 years of age, we used a more inclusive upper limit to account for varying pubertal development, ${ }^{19}$ so that children who had not yet reached their final height by 14 years of age would not be excluded.

Selection of some of the exposure terms was based on theoretical considerations discussed in the literature on early life risk factors for childhood overweight. ${ }^{20}$ In addition, inclusion criteria included any potential risk factors that had occurred prenatally, in the first year of life or when the child was 1 year of age. Studies that only included children with specific medical conditions or children from rare groups were excluded as these children may have a different set of risk factors and often are not comparable with the general population of children.

For anthropometric outcome, we used body mass index (BMI) as reported by the study author. BMI (adjusted for age and sex) is identified ${ }^{21}$ by the National Institute for Health and Clinical Excellence (NICE) guidelines as a preferred outcome measure in children aged 2 years or older. Overweight in childhood was defined as follows: by the International Obesity Task Force $(\mathrm{IOTF})^{18}$ as corresponding to an adult BMI $\geq 25$ or $\geq 30 \mathrm{~kg} / \mathrm{m}^{2}$; by the Centers for Disease Control and Prevention $(\mathrm{CDC})^{17}$ as a BMI $\geq 85$ th or $\geq 95$ th percentile; by the UK 1990 reference $^{22}$ as a $\mathrm{BMI} \geq 95$ th or $\geq 98$ th percentile; by the French reference $^{23}$ as a BMI $\geq 90$ th or $\geq 97$ th percentile; and by the German reference ${ }^{24}$ as a BMI $\geq 90$ th or $\geq 97$ th percentile. Studies that defined children as obese were considered to also include overweight children for the purpose of this review.

\section{Statistical analysis}

For certain risk factors that met meta-analysis eligibility criteria, the random effects model was utilised to pool the effect sizes of the individual risk factors taking into account both the sampling error and between-study heterogeneity. The $\mathrm{I}^{2}$ statistic was used to explain the between-study heterogeneity (0-100\%), with higher percentage variation suggesting more heterogeneity or differences among studies. The primary outcome was the adjusted odds of overweight. The meta-analysis only included adjusted outcomes (at least age and sex) to minimise confounding. Publication bias was assessed by an asymmetry test. ${ }^{25}$ The results were statistically significant when two-sided $p$ values were less than $5 \%$. All analyses were conducted in STATA V.11 (Stata Corporation, College Station, Texas, USA).

\section{RESULTS \\ Description of included studies}

Thirty prospective studies met the inclusion criteria. The most common reason for exclusion was the absence of an appropriate outcome for childhood overweight or obesity (figure 1). Many of these studies were nutrition or growth studies that used anthropometric outcomes but were not focused on childhood overweight or obesity. The second most common reason for exclusion was a non-prospective study design (figure 1). The key characteristics of the included studies are presented in table 1. The median age at follow-up was 6 years, ranging from 2 years ${ }^{26}$ to 14 years. ${ }^{27}$ Where reported, the median prevalence of childhood overweight was $13.2 \%$, ranging from $5.4 \%{ }^{28}$ to $29.6 \%{ }^{29}$ Ten of the 30 studies ${ }^{27} 30-38$ defined childhood overweight by IOTF cut-offs. ${ }^{18}$ Sixteen of the 30 studies ${ }^{28} 29$ 39-52 defined childhood overweight by CDC percentiles. ${ }^{17}$ Two UK studies ${ }^{53} 54$ defined childhood overweight by the UK 1990 growth reference centiles. $^{22}$ One study ${ }^{55}$ used reference data from France ${ }^{23}$ to define childhood overweight and another study ${ }^{26}$ used national reference data from Germany. ${ }^{24}$

\section{Maternal pre-pregnancy overweight}

Three studies ${ }^{29} 3354$ found a significant association between maternal pre-pregnancy overweight and subsequent childhood overweight. Hawkins et $a^{33}$ found that the children of mothers who were overweight before pregnancy were 1.37 times $(95 \%$ CI 1.18 to 1.58) more likely to be overweight at 3 years of age than children of normal weight parents. Reilly et a ${ }^{54}$ found that children of mothers who were obese before pregnancy were 4.25 times (95\% CI 2.86 to 6.32) more likely to be overweight at 7 years of age compared with children of non-obese mothers. Rooney et al ${ }^{29}$ found that children of mothers who were obese before pregnancy were 2.36 times (95\% CI 2.36 to 8.85) more at risk of being overweight between 9 and 14 years of age compared with children of non-obese mothers.

\section{Infant birth weight}

Seven studies 28293340424854 identified high birth weight as a potential risk factor for childhood overweight. The reported results were adjusted for maternal overweight status, sex and gestational weight gain. Overall, six of the seven studies 293340424854 found significant and strong positive associations between high birth weight and childhood overweight.

Three of the seven studies ${ }^{29} 4042$ reported the results in terms of birthweight categories and found significant associations. Dubois and Girard ${ }^{42}$ found that infants who weighed $\geq 4000 \mathrm{~g}$ at birth were 2.3 times (95\% CI 1.30 to 7.20 ) more likely to be overweight at 4.5 years of age compared with 


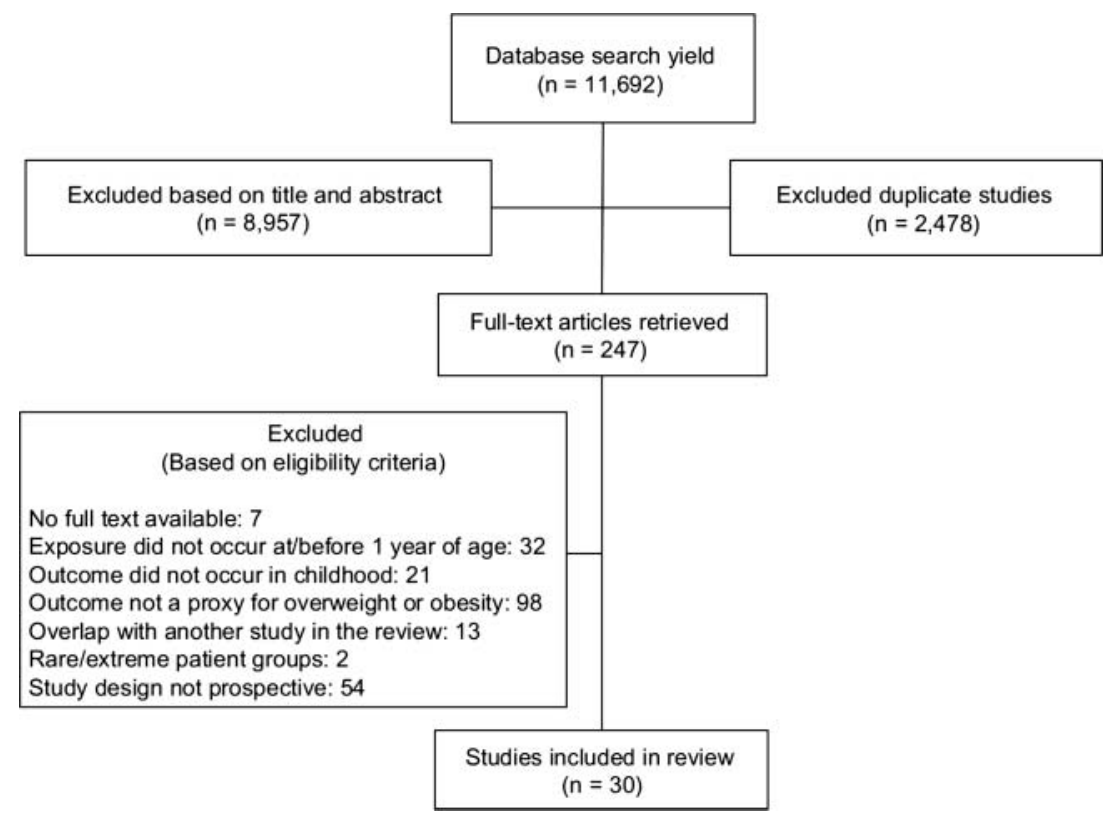

Figure 1 PRISMA flow chart of search strategy and selection process.

infants who weighed between 3000 and $4000 \mathrm{~g}$ at birth. Rooney et $a^{29}$ found that infants who weighed $\geq 8.5 \mathrm{lbs}$ $(3.86 \mathrm{~kg}$ ) were 2.17 times (95\% CI 1.22 to 3.87 ) more likely to be overweight between 4 and 5 years of age compared with infants who weighed between 7.01 and $8.49 \mathrm{lbs}(3.18-3.85 \mathrm{~kg})$. Ye et $a{ }^{40}$ found that infants who weighed $\geq 4.25 \mathrm{~kg}$ were 2.17 times (95\% CI 1.83 to 2.59 ) more likely to be overweight between 3 and 6 years of age compared with infants who weighed between 3 and $3.25 \mathrm{~kg}$.

Four of the seven studies ${ }^{28} 334854$ presented continuous exposures of birth weight ( $\mathrm{z}$ scores, grams). Hawkins et $a l^{33}$ found that for every 1 unit increase in birthweight $z$ score, the odds of being overweight at 3 years of age increased by 1.36 (95\% CI 1.30 to 1.42 ). Jones-Smith et al ${ }^{48}$ found that for every 1 unit increase in BMI $z$ score, the odds of overweight between 4 and 6 years of age increased dramatically by 7.62 (95\% CI 2.73 to 21.3). Reilly et $a t^{54}$ found that for every $100 \mathrm{~g}$ increase in birth weight, the odds of overweight at 7 years of age increased by 1.05 (95\% CI 1.03 to 1.08). The study by Stettler et $a 2^{28}$ was the only one to find no association between birth weight and childhood overweight.

\section{Infant rapid weight gain}

The six studies ${ }^{28} 2934424854$ that investigated infant rapid weight gain in the first year of life found significant associations with childhood overweight. Three studies ${ }^{29} 3442$ reported results for categorical exposures of early weight gain: comparing rapid growth to slow growth or normal growth. Dubois and Girard ${ }^{42}$ found that infants in the highest quintile of monthly weight gain from birth to 5 months were 3.9 (95\% CI 1.9 to 7.9) times more likely to be overweight at 4.5 years of age compared with infants in the lowest quintile of monthly weight gain. Hui et al ${ }^{34}$ compared accelerated growth (highest tertile) to slow growth (lowest tertile). In boys, there was a significant elevated odds of overweight at 7 years of age for infants who had accelerated growth regardless of birthweight group. In girls, there was a significant elevated odds of overweight at 7 years of age for medium and high birthweight infants who had accelerated growth. Rooney et $a^{29}$ found that being in the highest tertile of weight gain from birth to 5 months was associated with a 1.63 (95\% CI 1.05 to 2.53) times increased risk of overweight between 9 and 14 years of age.

Three of the six studies 284854 reported results for continuous exposures (BMI z score, grams per month) of early weight gain. Jones-Smith et $a l^{48}$ found that for every 1 unit increase in BMI $\mathrm{z}$ score from birth to 1 year, the odds of overweight between 4 and 6 years of age increased by 2.23 (95\% CI 1.12 to 4.46). Reilly et $a l^{54}$ found that for every $100 \mathrm{~g}$ per month in weight gain from birth to 1 year, the odds of overweight at 7 years of age increased by 1.06 (95\% CI 1.02 to 1.10). Stettler et $a l^{28}$ found that for every $100 \mathrm{~g}$ per month in weight gain from birth to 4 months, the odds of overweight at 7 years of age increased by 1.17 (95\% CI 1.11 to 1.24$)$.

\section{Maternal smoking during pregnancy}

Seven studies 32334142454954 investigated the impact of maternal smoking during pregnancy on childhood overweight. These studies met random effects meta-analysis criteria. The results in figure 2 show that children with mothers who had smoked regularly during pregnancy were $47 \%$ more likely to be overweight compared with children with mothers who had not smoked during pregnancy (adjusted odds ratio (AOR) 1.47, 95\% CI 1.26 to $1.73 ; \mathrm{I}^{2}=47.5 \% ; \mathrm{n}=7$ studies). There was a moderate but insignificant amount of heterogeneity $(p=0.064)$. Evidence of publication bias was detected by an asymmetry test $(p=0.001)$.

\section{Breastfeeding}

Ten studies $262733 \quad 363946 \quad 52-55$ compared breastfeeding with other types of feeding during the first year of life. Evidence for the protective effect of breastfeeding against overweight in childhood was mixed. Five studies ${ }^{33} 465253 \quad 55$ found that breastfeeding had significant protective effects against childhood overweight, while the other five studies $2627363954 \mathrm{did}$ not find significant associations. The studies met random effects meta-analysis criteria and a pooled AOR could therefore be obtained. Children who were 'ever breastfed' included those exclusively breastfed, ever breastfed or fed a mixture of formula and breast milk during the first year of life. The reference group 
Table 1 Key characteristics of included studies

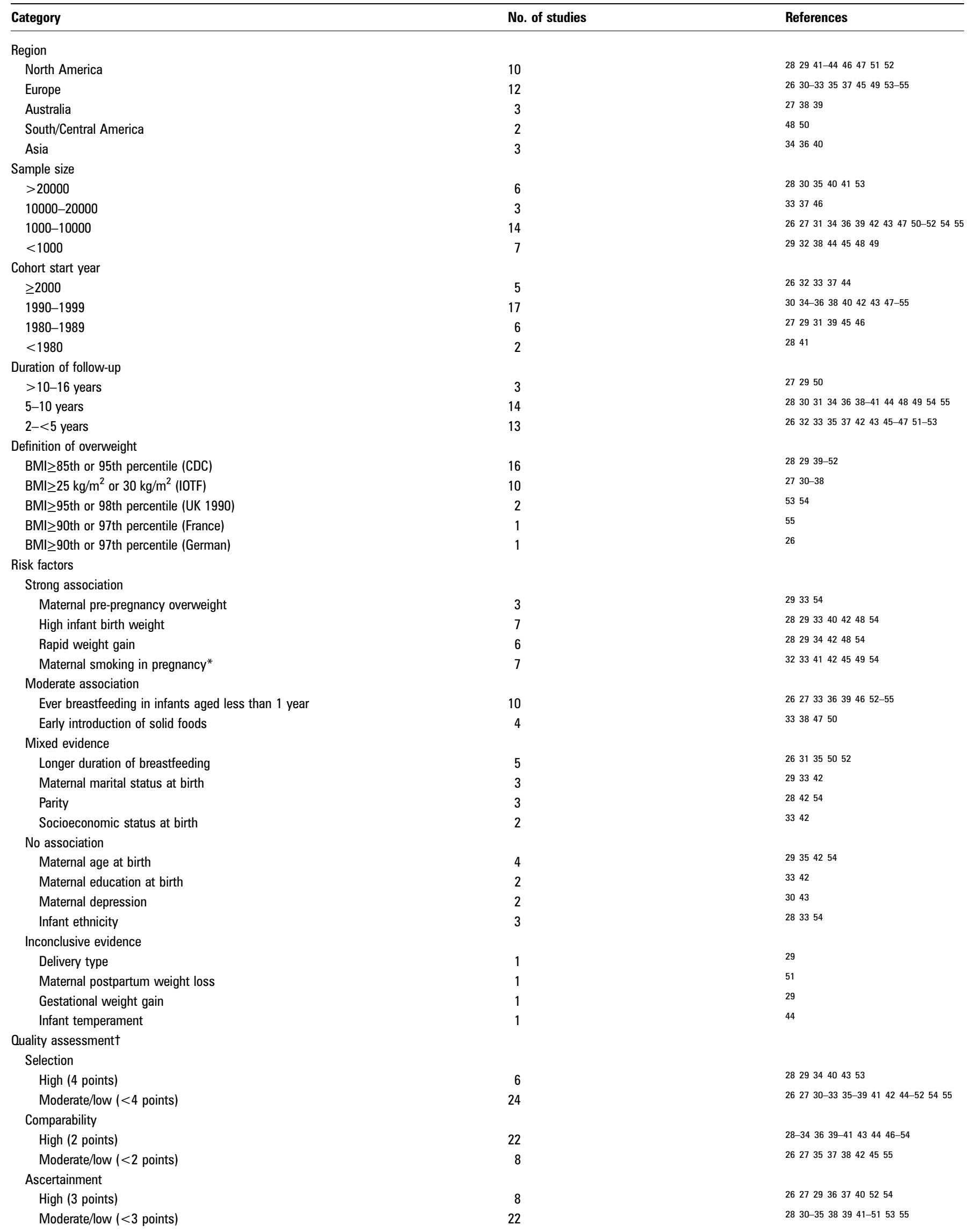

*Obtained from random-effects meta-analysis.

tNewcastle-Ottawa Scale.

BMI, body mass index; CDC, Centers for Disease Control and Prevention; IOTF, International Obesity Task Force. 


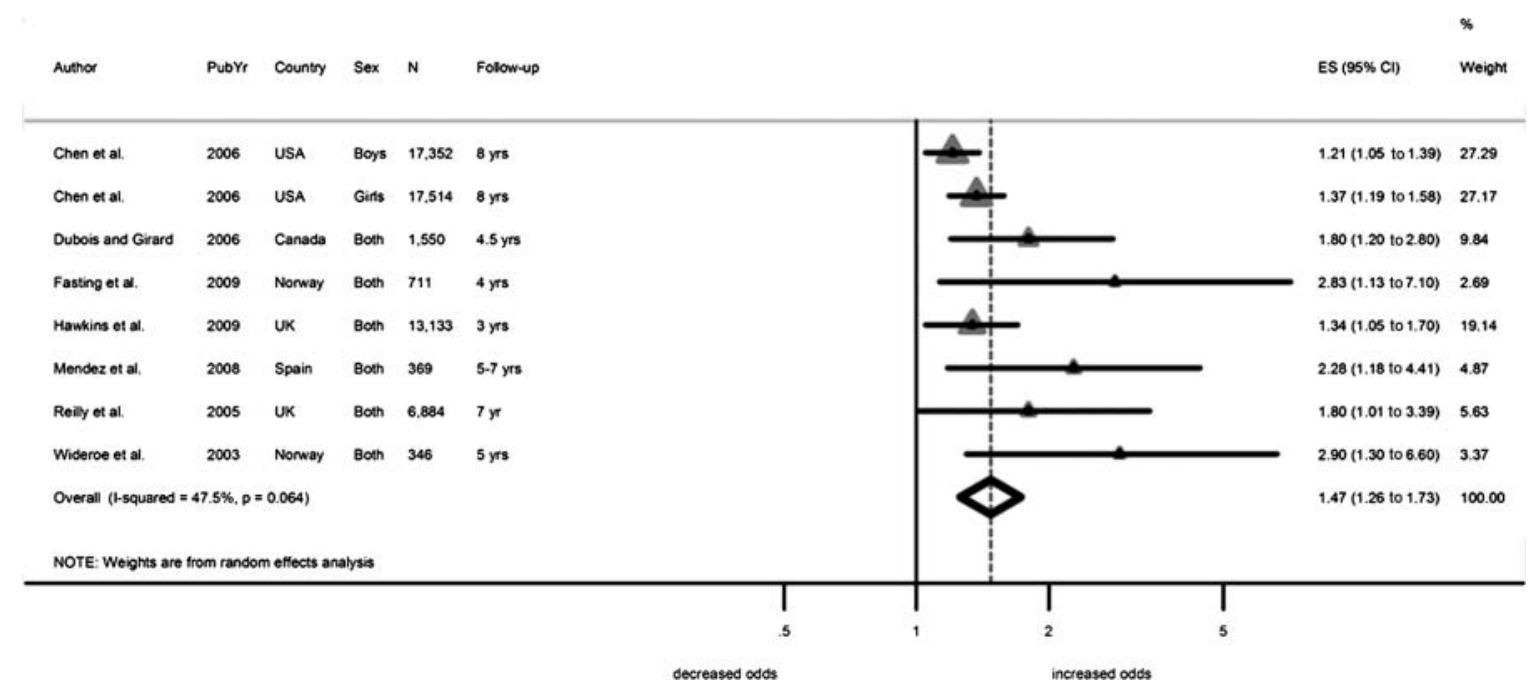

Figure 2 Pooled adjusted OR for childhood overweight from random effects meta-analysis of 7 studies: $^{32} 334142454954$ maternal smoking in pregnancy compared to no maternal smoking in pregnancy. ES, effect size.

of 'never breastfed' included children who were exclusively formula-fed. The results in figure 3 show that ever breastfeeding in the first year of life significantly decreased the odds of overweight in childhood by $15 \%$ (AOR $0.85,95 \%$ CI 0.74 to $0.99 ; \mathrm{I}^{2}=73.3 \% ; \mathrm{n}=10$ studies). There was no evidence of publication bias, which was confirmed by an asymmetry test $(p=0.248)$.

Five studies 2631355052 also analysed the impact of breastfeeding duration on childhood overweight in a subgroup of breastfed children. Four studies 31355052 did not find significant associations between the duration of breastfeeding and childhood overweight. However, Weyermann et al ${ }^{26}$ found a significant decrease in the odds of overweight at 2 years of age for infants who were breastfed for more than 6 months compared with infants who were breastfed for less than 3 months (AOR $0.4,95 \%$ CI 0.2 to 0.8 ).

\section{Introduction of solid foods}

Four studies ${ }^{33} 384750$ investigated the relationship between the earlier introduction of solid foods and childhood overweight.
There was some evidence supporting the early introduction of solid foods as a risk factor for later overweight. Hawkins et al ${ }^{33}$ found that infants given solid foods before 4 months were 1.12 times (95\% CI 1.02 to 1.23 ) more likely to be overweight at 3 years of age compared with infants who were given solid foods after 4 months. Huh et $a l^{47}$ found that formula-fed infants given solid foods before 4 months were 6.3 times $(95 \%$ CI 2.3 to 16.9) more likely to be overweight at 3 years of age compared with infants who were given solid foods between 4 and 5 months. However, this relationship was not significant in breastfed infants. Seach et $a l^{38}$ found that later introduction of solid foods was significantly associated with reducing overweight in childhood (AOR 0.91, 95\% CI 0.84 to 0.97). However, Neutzling et $a l^{50}$ did not find an association between the early introduction of solid foods and childhood overweight.

\section{Additional risk factors}

No independent association with childhood overweight was found for maternal age at birth, ${ }^{29} 354254$ maternal education at birth, ${ }^{33}$ maternal antenatal or postnatal depression ${ }^{30} 43$ or

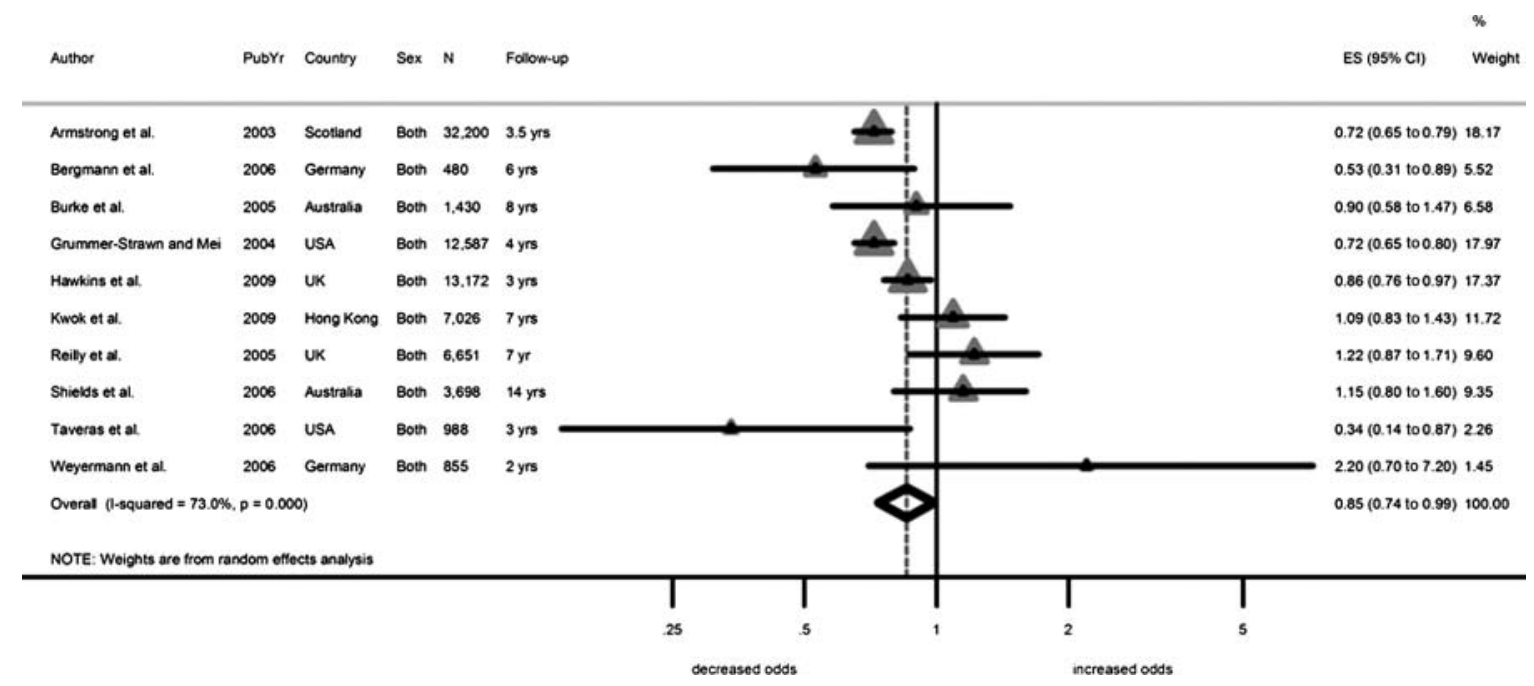

Figure 3 Pooled adjusted OR for childhood overweight from random effects meta-analysis of 10 studies: ${ }^{26} 273336394652-55$ : ever breastfed compared with never breastfed. ES, effect size. 
infant ethnicity. ${ }^{28} 3354$ Due to the limited number of studies, there was inconclusive evidence for the following factors: delivery type, ${ }^{29}$ maternal postpartum weight loss, ${ }^{51}$ gestational weight gain ${ }^{29}$ and 'fussy' infant temperament. ${ }^{44}$ There was also conflicting evidence for the following factors: maternal marital status at birth, ${ }^{29} 3342$ socioeconomic status at birth ${ }^{33} 42$ and parity. ${ }^{28} 4254$

\section{Quality assessment}

Table 1 shows that both 'selection' and 'ascertainment' quality assessment scores were weak among the studies reviewed, while 'comparability' was strong. Only six studies were considered to have high 'selection' quality. This was due to the fact that most studies collected data using self-reported surveys completed by parents. Self-reported surveys are subject to recall bias, and variables such as breastfeeding status or child anthropometry measurements were not validated by HCPs. The six studies that did obtain a high 'selection' score utilised HCPs or health visitors to validate the data through structured interviews in the home or clinic. Similarly, only eight studies were considered to have high 'ascertainment'. Attrition bias is a potential issue with many of the observation studies reviewed. To achieve a high 'ascertainment' score, studies were required to have $\geq 80 \%$ follow-up rates or descriptions of those lost if there was $<80 \%$ follow-up. The majority of the studies did not describe participants lost to follow-up and therefore, we could not determine if those lost influenced the overall results of the study.

\section{DISCUSSION}

\section{Explanation of findings}

This systematic review of 30 prospective studies identified several significant early life risk factors for childhood overweight: maternal pre-pregnancy overweight, high infant birth weight, early infant rapid weight gain and maternal smoking during pregnancy. There was a moderate protective effect of ever breastfeeding during the first year on subsequent childhood overweight. There was some evidence that the early introduction of solid foods was associated with childhood overweight, and mixed evidence for longer duration of breastfeeding during the first year, maternal marital status at birth and parity. There was no association for the remaining factors or the evidence was inconclusive due to a lack of studies.

The studies exploring maternal pre-pregnancy overweight as a risk factor found higher odds of overweight in offspring who had parents who were classified as overweight or obese. In an early systematic review of childhood predictors of adult obesity by Parsons et al, ${ }^{20}$ a strong positive correlation between offspring anthropometry and parental anthropometry was identified. Parsons et $a l^{20}$ suggest that environment strongly influences genetic components such as genetic predisposition to select fatty foods or inability to perform physical activity. In addition, some genes involved in homeostatic regulation, appetite suppression and maintaining energy balance have been linked to well-known obesity models. ${ }^{56}$ It is likely that the relationship between parental and childhood overweight is influenced by both genes and lifestyle. However, it is not clear what these mechanisms are or how the environment overrides psychological appetite controls and homeostatic body weight regulation. ${ }^{56}$

The hereditary components of parental overweight may also influence infant anthropometry. Oken et a ${ }^{57}$ found in a review of the infant growth literature that parental adiposity is directly associated with offspring birth weight. In other systematic reviews ${ }^{58} 59$ of infant size and obesity, the majority of studies consistently found that infants who were heavier during infancy were more likely to develop obesity in childhood, adolescence and adulthood. In our review, higher birth weight was significantly associated with later childhood overweight in the majority of studies. Additionally, we found that early rapid weight gain was associated with childhood overweight, as has been previously reported in three earlier systematic reviews. ${ }^{60-62}$ There are two possible mechanisms whereby rapid infancy weight gain could lead to later obesity. Druet et $a l^{60}$ suggest that rapid weight gain in infancy could be due to nutrition and the environment, or rapid weight gain may be a genetic marker of the future trajectory of weight gain. Evidence also suggests that birth weight may modify genetic susceptibility towards weight gain and future obesity risk. ${ }^{63}$

Modifying nutritional factors connected with parental feeding behaviours in infancy are primarily associated with breastfeeding and weaning. We found in a meta-analysis of 10 prospective studies that breastfeeding anytime in the first year life reduced the adjusted odds of overweight in childhood by $15 \%$ compared with not breastfeeding. This result is similar to that of a meta-analysis of nine observational studies conducted by Arenz et al ${ }^{64}$ which found that breastfeeding reduced the adjusted odds of obesity in childhood by $22 \%$ compared with formula feeding. Possible biological explanations include higher plasma insulin levels in formula-fed infants that could stimulate fat deposition and bioactive factors in breast milk which might modulate growth.

This review found some evidence that the early introduction of solid foods had an impact on childhood overweight. However, breastfeeding possibly confounded the relationship between age at weaning and childhood overweight because no association was found in breastfed infants. Wasser et al ${ }^{65}$ found in a cross-sectional study of 217 mothers, that infants who were perceived as 'fussy' eaters were more likely to receive complementary foods before 4 months of age. Thus, infant temperament (soothability by food, 'fussy' eating behaviour) may have a confounding effect on the relationship between weaning and childhood overweight.

Lifestyle behaviours such as maternal smoking in pregnancy, were found to be significantly associated with childhood overweight. We found in a meta-analysis of seven prospective studies that maternal smoking in pregnancy increased the adjusted odds of childhood overweight by $47 \%$. This result was similar to that of a previous meta-analysis of 14 observational studies by Oken et al ${ }^{66}$ where maternal smoking in pregnancy increased the adjusted odds of overweight by $50 \%$. Exposure to cigarette smoke increases the risk of fetal adverse development and growth restriction. Even though maternal smoking in pregnancy may result in growth restriction in utero, some studies have found that affected infants exhibit extremely high rapid postnatal weight gain. ${ }^{67} 68$ It is also likely that maternal smoking in pregnancy is a proxy for other social and lifestyle characteristics. In a US study of low income children and their parents, children of smokers were found to have poor diet quality with high levels of saturated fat, high levels of cholesterol intake and low levels of fibre intake. ${ }^{69}$

\section{Clinical implications}

Unlike prior reviews, we have comprehensively investigated the risk factors for childhood overweight in the first year of life using only prospective studies. Our review included both overweight and obesity outcomes in contrast to previous reviews which mostly investigated obesity alone. Previous 
comprehensive reviews ${ }^{20} 70$ have studied childhood predictors with adult obesity as the endpoint but have not fully investigated infant factors. Other reviews on breastfeeding, ${ }^{64}$ infant growth, ${ }^{60}$ hereditary factors ${ }^{57-59}$ and maternal smoking ${ }^{66}$ have only focused on these specific areas. In our study we have also reviewed factors in the first year of life that have not previously been fully examined: parity, socioeconomic status, maternal education, maternal depression, infant ethnicity, delivery type, maternal postpartum weight loss, gestational weight gain and infant temperament. In addition, most previous reviews incorporated observational evidence. As the use of only prospective studies in this study ensures excellent observational evidence, ${ }^{71}$ the findings could be used to develop screening guidelines or checklists for HCPs for identifying infants at greatest risk. Most of the identified risk factors are static (non-modifiable), but potentially dynamic (modifiable) risk factors such as maternal response to infant temperament, could be explored further with a contemporary cohort of children. However, recent research ${ }^{72}$ has found that using a purely statistical approach for developing a risk factor checklist from birth cohort data may not provide acceptable levels of specificity and sensitivity in a clinical setting, and so clinical input is needed from practitioners, service providers and parent groups. ${ }^{72}$ In addition, there are many practical and ethical considerations including the acceptability of such a checklist by parents and the feasibility of implementing it in the existing health service. Any risk factor checklist requires testing in the field and needs to be accompanied by clinical guidance for HCPs on how to approach parents of infants identified as being at risk, which should be supported by evidence-based intervention.

\section{Limitations}

One limitation was that we used a late age cut-off of 16 years to account for varying pubertal development. This may have meant that some children close to their final height were included. However, the longest duration of follow-up in all included studies was 14 years (only one study) with a median of 6 years. Most children included in our review were under 10 years of age and had not yet reached their final height. Thus, the effects of using a more inclusive age cut-off were marginal. Another limitation was the use of BMI as an outcome measure. This was a clinical recommendation due to the practicality and efficiency of BMI monitoring for HCPs as supported by NICE guidance. ${ }^{21}$ However BMI is an indirect measurement of adiposity, in contrast to skin fold and body fat percentage measurements which are direct measurements of subcutaneous, central or total adiposity. While BMI is highly correlated with direct measurements of adiposity, it is also influenced by lean body and bone mass.

Additionally, the cohort studies examined in this review included samples of children from a range of different socioeconomic and cultural backgrounds. The findings may need to be interpreted with caution since there is some evidence that BMI systematically underestimates adiposity in South Asian subjects and overestimates adiposity in Black African Caribbean subjects because of its association with height. ${ }^{73}$

There was also a great deal of heterogeneity between overweight outcomes in childhood depending on the particular growth reference data used (IOTF, CDC, UK 1990, French, German). This is likely to have influenced the overweight prevalence depending on the definitions that the authors used in their respective studies. Therefore, we could only summarise results based on directional associations between groups of exposures and the childhood overweight outcome for several potential risk factors. Finally, there is evidence of publication bias in the meta-analysis of seven smoking-related studies. However, we adjusted the pooled OR using 'trim and fill' by simulating a symmetrical set of studies, which yielded a similar AOR of 1.34 ( $95 \%$ CI 1.13 to $1.60 ; I^{2}=53.2 \% ; n=12$ studies).

\section{CONCLUSION}

This systematic review identified several early life risk factors for childhood overweight and provides high quality evidence that could be used by HCPs to identify infants at greatest risk. We found strong evidence that early rapid weight gain, high birth weight, maternal pre-pregnancy overweight and maternal smoking in pregnancy increased the likelihood of childhood overweight. There was also a moderate protective effect of breastfeeding on childhood overweight. There was some evidence to suggest that the early introduction of solid foods was associated with childhood overweight. Several factors were found to have mixed, inconclusive or no association with childhood overweight as follows: breastfeeding duration, maternal marital status, parity, socioeconomic status, maternal age, maternal education, maternal depression, infant ethnicity, delivery type, maternal postpartum weight loss, gestational weight gain and infant temperament. Our future research will focus on exploring the validity and feasibility of identifying infants at risk of developing childhood overweight in clinical practice.

Acknowledgements We would like to thank Rachel Illingworth for supporting this work. We would also like to thank Dr Dilip Nathan, Nottingham University Hospitals Trust and Professor Aloysius Niro Siriwardena for their contributions to this work.

Contributors SFW, SAR, MY, CPG and JAS developed the study design, carried out the systematic review and conducted the data analysis. SFW prepared the first draft of the manuscript. SFW, SAR, MY and CG revised the first draft and prepared the final version of the manuscript. All study authors approved the final version of the manuscript.

Funding This work was funded by NHS Nottinghamshire County PCT.

\section{Competing interests None.}

Provenance and peer review Not commissioned; externally peer reviewed.

\section{REFERENCES}

1. National Health Service. Statistics on obesity, physical activity and diet-England 2010. National Health Service. NHS Information Centre: The Health and Social Care Information Centre, Leeds, 2010.

2. Department of Health. National Child Measurement Programme: England, 2009/10 school year. Department of Health: The Health and Social Care Information Centre, London, 2010.

3. Gardner DS, Hosking J, Metcalf BS, et al. Contribution of early weight gain to childhood overweight and metabolic health: a longitudinal study (EarlyBird 36) Pediatrics 2009;123:e67-73.

4. Dietz WH. Health consequences of obesity in youth: childhood predictors of adult disease. Pediatrics 1998;101(Suppl 2):518-25.

5. Dixon JB. The effect of obesity on health outcomes. Mol Cell Endocrinol 316:104-8.

6. Anzman SL, Rollins BY, Birch LL. Parental influence on children's early eating environments and obesity risk: implications for prevention. Int $J$ Obes 2010;34:1116-4.

7. Johnston BD, Huebner CE, Anderson ML, et al. Healthy steps in an integrated delivery system: child and parent outcomes at 30 months. Arch Pediatr Adolesc Med 2006;160:793-800.

8. Talvia S, Rasanen L, Lagstrom $\mathrm{H}$, et al. Longitudinal trends in consumption of vegetables and fruit in Finnish children in an atherosclerosis prevention study (STRIP). Eur J Clin Nutr 2006;60:172-80.

9. Johnson Z, Molloy B, Scallan E, et al. Community mothers programme - seven year follow-up of a randomized controlled trial of non-professional intervention in parenting. J Pub Health 2000;22:337-42.

10. Horodynski MA, Stommel M. Nutrition education aimed at toddlers: an intervention study. Pediatr Nurs 2005;31:367-72. 
11. Promoting optimal monitoring of child growth in Canada: using the new World Health Organization growth charts—executive summary. Paediatr Child Health 2010;15:77-83.

12. Barlow SE, Committee atE. Expert committee recommendations regarding the prevention, assessment, and treatment of child and adolescent overweight and obesity: summary report. Pediatrics 2007;120(Suppl 4):S164-92.

13. Institute of Medicine. Early childhood obesity prevention policies. Washington DC: Institute of Medicine (loM), 2011

14. McCormick DP, Sarpong K, Jordan L, et al. Infant obesity: are we ready to make this diagnosis? J Pediatr 2010;157:15-19.

15. Redsell SA, Atkinson P, Nathan D, et al. Preventing childhood obesity during infancy in UK primary care: a mixed-methods study of HCPs' knowledge, beliefs and practice. BMC Family Practice 2011:12:54.

16. Wells G, Shea B, O'Connel D, et al. The Newcastle-Ottawa Scale (NOS) for assessing the quality of nonrandomised studies in meta-analysis. 2008 Date;Volume. http://www. ohri.ca/programs/clinical epidemiology/oxford.asp (accessed 6 Aug 2011).

17. CDC. CDC growth charts for the United States: methods and development. Vital Health Stat 2000;8:42.

18. Cole TJ, Bellizzi MC, Flegal KM, et al. Establishing a standard definition for child overweight and obesity worldwide: international survey. Br Med J 2000;320:1240.

19. Dietz WH. Periods of risk in childhood for the development of adult obesity — what do we need to learn? J Nutr 1997;127:1884S-6S.

20. Parsons TJ, Power C, Logan S, et al. Childhood predictors of adult obesity: a systematic review. Int J Obes Relat Metab Disord 1999;23(Suppl 8):S1-107.

21. Obesity guidance on the prevention, identification, assessment and management of overweight and obesity in adults and children. NICE clinical guideline 43. London: National Institute for Health and Clinical Excellence, 2006.

22. Cole TJ, Freeman JV, Preece MA. British 1990 growth reference centiles for weight, height, body mass index and head circumference fitted by maximum penalized likelihood. Stat Med 1998:17:407-29.

23. Rolland-Cachera MF, Cole TJ, Sempe M, et al. Body Mass Index variations: centiles from birth to 87 years. Eur J Clin Nutr 1991:45:13-21.

24. Kromeyer-Hauschild K, Wabitsch M, Kunze D, et al. Perzentile für den Body-mass-Index für das Kindes- und Jugendalter unter Heranziehung verschiedener deutscher Stichproben. Monatsschrift Kinderheilkunde 2001;149:807-18.

25. Egger M, Davey Smith G, Schneider M, et al. Bias in meta-analysis detected by a simple, graphical test. Br Med J 1997;315:629-34.

26. Weyermann M, Rothenbacher $\mathrm{D}$, Brenner $\mathrm{H}$. Duration of breastfeeding and risk of overweight in childhood: a prospective birth cohort study from Germany. Int $\mathrm{J}$ Obes 2006; 30:1281-7

27. Shields $\mathbf{L}, \mathrm{O}^{\prime}$ Callaghan $\mathrm{M}$, Williams GM, et al. Breastfeeding and obesity at 14 years: a cohort study. J Paediatr Child Health 2006;42:289-96.

28. Stettler N, Zemel BS, Kumanyika S, et al. Infant weight gain and childhood overweight status in a multicenter, cohort study. Pediatrics 2002;109:194-9.

29. Rooney BL, Mathiason MA, Schauberger CW. Predictors of Obesity in Childhood, Adolescence, and Adulthood in a Birth Cohort. Matern Child Health J 2010;7:7

30. Ajslev TA, Andersen CS, Ingstrup KG, et al. Maternal postpartum distress and childhood overweight. PLoS One 2010;5:e11136.

31. Buyken AE, Karaolis-Danckert N, Remer T, et al. Effects of breastfeeding on trajectories of body fat and BMl throughout childhood. Obesity 2008;16:389-95.

32. Fasting $\mathbf{M H}$, Oien T, Storro 0 , et al. Maternal smoking cessation in early pregnancy and offspring weight status at four years of age. A prospective birth cohort study. Early Hum Dev 2009;85:19-24.

33. Hawkins SS, Cole TJ, Law C, et al. An ecological systems approach to examining risk factors for early childhood overweight: findings from the UK Millennium Cohort Study. J Epidemiol Community Health 2009:63:147-55.

34. Hui LL, Schooling CM, Leung SS, et al. Birth weight, infant growth, and childhood body mass index: Hong Kong's children of 1997 birth cohort. Neurology 2008; 70:788-94.

35. Huus K, Ludvigsson JF, Enskar K, et al. Exclusive breastfeeding of Swedish children and its possible influence on the development of obesity: a prospective cohort study. BMC Pediatrics 2008;8:42

36. Kwok MK, Schooling CM, Lam TH, et al. Does breastfeeding protect against childhood overweight? Hong Kong's 'Children of 1997' birth cohort. Int J Epidemiol 2010:39:297-305.

37. Pearce A, Li L, Abbas J, et al. Is childcare associated with the risk of overweight and obesity in the early years? Findings from the UK Millennium Cohort Study. Int $J$ Obes 2010;34:1160-8.

38. Seach KA, Dharmage SC, Lowe AJ, Dixon JB. Delayed introduction of solid feeding reduces child overweight and obesity at 10 years. Int J Obes 2010;34:1475-9.

39. Burke V, Beilin LJ, Simmer K, et al. Breastfeeding and overweight: longitudinal analysis in an Australian birth cohort. J Pediatr 2005:147:56-61.

40. Ye R, Pei L, Ren A, et al. Birth weight, maternal body mass index, and early childhood growth: a prospective birth cohort study in China. J Epidemiol 2010;20:421-8.

41. Chen A, Pennell ML, Klebanoff MA, et al. Maternal smoking during pregnancy in relation to child overweight: follow-up to age 8 years. Int J Epidemiol 2006:35:121-30.
42. Dubois L, Girard M. Early determinants of overweight at 4.5 years in a population-based longitudinal study. Int J Obes 2006;30:610-17.

43. Ertel KA, Koenen KC, Rich-Edwards JW, et al. Antenatal and postpartum depressive symptoms are differentially associated with early childhood weight and adiposity. Paediatr Perin Epidemiol 2010;24:179-89.

44. Faith MS, Hittner JB. Infant temperament and eating style predict change in standardised weight status and obesity risk at 6 years of age. Int $\mathrm{J}$ Obes 2010;34:1515-3.

45. Wideroe M, Vik T, Jacobsen G, et al. Does maternal smoking during pregnancy cause childhood overweight? Paediatr Perin Epidemiol 2003;17:171-9.

46. Grummer-Strawn LM, Mei ZG. Does breastfeeding protect against pediatric overweight? Analysis of longitudinal data from the Centers for Disease Control and Prevention Pediatric Nutrition Surveillance System. Pediatrics 2004; 113:e81-6.

47. Huh SY, Rifas-Shiman SL, Taveras EM, et al. Timing of solid food introduction and risk of obesity in preschool-aged children. Pediatrics 2011;127:e544-51.

48. Jones-Smith JC, Fernald LC, Neufeld LM. Birth size and accelerated growth during infancy are associated with increased odds of childhood overweight in Mexican children. Epidemiology 2007;18:722-9.

49. Mendez MA, Torrent M, Ferrer $\mathrm{C}$, et al. Maternal smoking very early in pregnancy is related to child overweight at age 5-7 y. Am J Clin Nutr 2008;87:1906-13.

50. Neutzling M, Hallal PC, Pavin Araujo CL, et al. Infant feeding and obesity at 11 years: Prospective birth cohort study. Int J Pediatr Obes 2009;4:143-9.

51. Sonneville KR, Rifas-Shiman SL, Oken E, et al. Longitudinal association of maternal attempt to lose weight during the postpartum period and child obesity at age 3 years. Obesity 2011:24:24.

52. Taveras EM, Rifas-Shiman SL, Scanlon KS, et al. To what extent is the protective effect of breastfeeding on future overweight explained by decreased maternal feeding restriction? Pediatrics 2006;118:2341-8.

53. Armstrong J, Reilly JJ. Breastfeeding and lowering the risk of childhood obesity. Lancet 2002;359:2003-4.

54. Reilly JJ, Armstrong J, Dorosty AR, et al. Early life risk factors for obesity in childhood: cohort study. Br Med J (Clinical Research Edition) 2005:330:1357-9.

55. Bergmann KE, Bergmann RL, Von Kries R, et al. Early determinants of childhood overweight and adiposity in a birth cohort study: role of breast-feeding. Behav Modif 2003;27:54-67.

56. Lenard NR, Berthoud HR. Central and peripheral regulation of food intake and physical activity: pathways and genes. Obesity 2008;16:S11-22.

57. Oken E, Gillman MW. Fetal origins of obesity. Obes Res 2003;11:496-506.

58. Baird J, Fisher D, Lucas $\mathrm{P}$, et al. Being big or growing fast: systematic review of size and growth in infancy and later obesity. Br Med J 2005;331:929.

59. Yu ZB, Han SP, Zhu GZ, et al. Birth weight and subsequent risk of obesity: a systematic review and meta-analysis. Obes Rev 2011;12:525-42.

60. Druet C, Stettler N, Sharp S, et al. Prediction of childhood obesity by infancy weight gain: an individual-level meta-analysis. Paediatr Perin Epidemiol 2011:26:19-26

61. Monteiro PO, Victora CG. Rapid growth in infancy and childhood and obesity in later life-a systematic review. Obes Rev 2005:6:143-54.

62. Ong KK, Loos RJF. Rapid infancy weight gain and subsequent obesity: Systematic reviews and hopeful suggestions. Acta Paediatrica 2006;95:904-8.

63. Hong J, Shi J, Oi L, et al. Genetic susceptibility, birth weight and obesity risk in young Chinese. Int $J$ Obes 2012.

64. Arenz S, Ruckerl R, Koletzko B, et al. Breast-feeding and childhood obesity[mdash]a systematic review. Int J Obes Relat Metab Disord 2004;28:1247-56.

65. Wasser H, Bentley M, Borja J, et al. Infants perceived as "Fussy" are more likely to receive complementary foods before 4 months. Pediatrics 2011;127:229-37.

66. Oken E, Levitan EB, Gillman MW. Maternal smoking during pregnancy and child overweight: systematic review and meta-analysis. Int J Obes 2008;32:201-10.

67. Jacobson JL, Jacobson SW, Sokol RJ. Effects of prenatal exposure to alcohol, smoking, and illicit drugs on postpartum somatic growth. Alcohol Clin Exp Res 1994; 18:317-23.

68. Karaolis-Danckert N, Buyken AE, Kulig M, et al. How pre- and postnatal risk factors modify the effect of rapid weight gain in infancy and early childhood on subsequent fat mass development: results from the Multicenter Allergy Study 90. Am J Clin Nutr 2008;87:1356-64

69. Johnson RK, Wang MQ, Smith MJ, et al. The association between parental smoking and the diet quality of low-income children. Pediatrics 1996;97:312-17.

70. Singh AS, Mulder C, Twisk JW, et al. Tracking of childhood overweight into adulthood: a systematic review of the literature. Obes Rev 2008:9:474-88.

71. Tacconelli E. Systematic reviews: CRD's guidance for undertaking reviews in health care. Lancet Infect Dis 2010:10:226.

72. Levine RS, Dahly DL, Rudolf MC. Identifying infants at risk of becoming obese: can we and should we? Public Health 2011:126:123-8.

73. Nightingale CM, Rudnicka AR, Owen CG, et al. Patterns of body size and adiposity among UK children of South Asian, black African-Caribbean and white European origin: child heart and health Study in England (CHASE Study). Int J Epidemiol 2010:40:33-44. 